\title{
Rare pulmonary disease and orphan drugs: a path to the future
}

\author{
Sergio Harari (1) ${ }^{1}$, Yochai Adir ${ }^{2}$ and Marc Humbert $\mathbb{1}^{3,4,5}$
}

\begin{abstract}
Affiliations: ${ }^{1}$ U.O. di Pneumologia e Terapia Semi-Intensiva Respiratoria, Servizio di Fisiopatologia Respiratoria e Emodinamica Polmonare, Ospedale San Giuseppe, MultiMedica IRCCS, Milan, Italy. ${ }^{2}$ Pulmonary Division, Lady Davis Carmel Medical Center, Faculty of Medicine, The Technion Institute of Technology, Haifa, Israel. ${ }^{3}$ Service de Pneumologie, Hôpital Bicêtre, AP-HP, Le Kremlin-Bicêtre, France. ${ }^{4}$ INSERM UMR S 999 , Hôpital Marie Lannelongue, Le Plessis Robinson, France. ${ }^{5}$ Université Paris-Sud, Faculté de Médecine, Université Paris-Saclay, Le Kremlin-Bicêtre, France.
\end{abstract}

Correspondence: Sergio Harari, U.O. di Pneumologia e Terapia Semi-Intensiva Respiratoria, Servizio di Fisiopatologia Respiratoria ed Emodinamica Polmonare, Ospedale San Giuseppe, MultiMedica IRCCS, via San Vittore 12, 20123 Milan, Italy. E-mail: sergiodsergioharari.it

@ERSpublications

The interest in rare diseases is growing. The progress in this field has been made possible by a coordinated action between respiratory researchers, health authorities and patients associations. http://bit.ly/2kIurPH

Cite this article as: Harari S, Adir Y, Humbert M. Rare pulmonary disease and orphan drugs: a path to the future. Eur Respir Rev 2019; 28: 190115 [https://doi.org/10.1183/16000617.0115-2019].

In this issue of the European Respiratory Review (ERR), we are delighted to publish a series of articles on rare pulmonary diseases discussing hot topics presented at the 8th International Meeting on Pulmonary Rare Diseases and Orphan Drugs, which took place in Milan, Italy in March 2019. The meeting was endorsed by the European Respiratory Society and the European Reference Network - LUNG; a network of healthcare providers dedicated to the care and investigation of patients suffering from rare respiratory diseases approved by the European Commission's Board of Member States in December 2016 (https://ec. europa.eu/health/ern/networks_en) [1].

This international meeting, which is held every 2 years, is entirely dedicated to the management of rare pulmonary parenchymal and vascular diseases and rare variants of more common respiratory conditions (such as severe refractory asthma and pulmonary complications of particularly common therapies). In recent years, the interest for rare diseases has grown in the entire medical community, and many respiratory physicians were pioneers in this field of medicine. The tremendous progress has been made possible by several factors, including a more appropriate classification (i.e. pulmonary hypertension and idiopathic pulmonary fibrosis (IPF)), the collective effort of research by the respiratory medicine community and progress in terminology of rare diseases by Orphanet (www.orpha.net). Important factors for the success of this fight were also: the emergence of coordinated action against rare diseases by health authorities in several western countries and at the European level; the role of patient associations, which raised awareness, funding and, in some cases, facilitated clinical trials (as in the case of lymphangioleiomyomatosis for the MILES trial); and investment by major pharmaceutical companies, itself fostered by orphan drug legislation [2].

However, by far the paradigm of the research in this field and in the development of orphan drugs is pulmonary artery hypertension; this disease has greatly benefited from the establishment of an international network of research focused on understanding the complex pathogenesis and development of

Provenance: Commissioned article, peer reviewed.

Received: 05 Sept 2019 | Accepted: 12 Sept 2019

Copyright $\odot$ ERS 2019. This article is open access and distributed under the terms of the Creative Commons Attribution Non-Commercial Licence 4.0. 
the illness [2]. The registration of more than 10 different drugs, with important impact on outcomes as well as quality of life of patients suffering from this disease, witnesses the strong effort made in the last 20 years to help clinicians, patients and relatives fighting this devastating pulmonary vascular disease.

In recent years, the ERR has published several articles on rare pulmonary diseases and orphan drugs. The publication of these reports from the 8th International Meeting on Rare Pulmonary Diseases and Orphan Drugs goes further in this direction. Furthermore, a new series on rare genetic interstitial lung disorders will be published in the coming months. We believe that rare diseases represent an important field of medicine not only for those pulmonologists who are skilled in diagnosing and treating particular groups of these illnesses, but also for all respiratory physicians. Sharing knowledge and exchanging updates among experts on different areas of clinical and basic research in respiratory medicine, with different specialised pulmonary medicine expertise, from vascular to interstitial lung diseases, is part of the stimulating challenge of providing new insights into science and clinical care, thus helping patients and supporting doctors [3].

The proceedings from this meeting includes several review articles that focus on rare pulmonary diseases written by outstanding experts, providing an update on new issues and difficult problems in the management of those illnesses. Different aspects of IPF are discussed, starting from what we learnt from basic science. YANAGIHARA et al. [4] have written an excellent state-of-the-art review on our knowledge of this disease. BorIE et al. [5] provide a very clear and updated overview on the genetics of interstitial lung disease, which helps understand when to suspect genetic abnormalities and when to ask for genetic screening. The therapy of IPF and future perspectives are presented by SomogYi et al. [6], while Coluins and RAGHU [7] present the very hot topic of antifibrotic treatments for non-IPF fibrotic interstitial lung disease, which is also discussed in the editorial from Cotrin [8]. Finally, Caminati et al. [9] deepen the important issue of the clinical impact and management of comorbidities in IPF patients, who are often old and with a history of smoking.

Pulmonary hypertension is the other major topic with WEATHERALD et al. [10] providing an overview of pulmonary artery hypertension screening in systemic sclerosis, while Elia et al. [11] debate the difficult issue of looking at future perspectives of pulmonary hypertension in chronic lung disease. Finally, CADRANEl et al. [12] face an increasingly new problem, pulmonary complications of immune checkpoint inhibitors and present it in the context of nonsmall cell lung cancer.

We thank the ERR for hosting these selected articles and hope the readers will enjoy them. The slide presentations from the 8th International Meeting on Pulmonary Rare Diseases and Orphan Drugs are available from www.pulmonaryrarediseases.com.

Conflict of interest: S. Harari reports grants and personal fees from Roche, Actelion and Boehringer Ingelheim, outside the submitted work. Y. Adir reports grants and personal fees from Actelion and GSK, and personal fees from Sanofi, Merck, United Therapeutics, BI and AstraZeneca, outside the submitted work. M. Humbert reports personal fees from Actelion and Merck, grants and personal fees from Bayer, GSK and United Therapeutics, outside the submitted work.

\section{References}

1 Humbert M, Wagner TO. Rare respiratory diseases are ready for primetime: from Rare Disease Day to the European Reference Networks. Eur Respir J 2017; 49: 1700085.

2 Harari S, Cottin V, Humbert M. Global effort against rare and orphan diseases. Eur Respir Rev 2012; 21: 1-2.

3 Harari S, Humbert M. Toward better management of rare and orphan pulmonary diseases. Eur Respir J 2016; 47: 1334-1133.

4 Yanagihara T, Sato S, Upagupta C, et al. What have we learned from basic science studies on idiopathic pulmonary fibrosis? Eur Respir Rev 2019; 28: 190029.

5 Borie R, Le Guen P, Ghanem M, et al. The genetics of interstitial lung diseases. Eur Respir Rev 2019; 28 : 190053.

6 Somogyi V, Chaudhuri N, Torrisi SE, et al. The therapy of idiopathic pulmonary fibrosis: what is next? Eur Respir Rev 2019; 28: 190021.

7 Collins BF, Raghu G. Antifibrotic therapy for fibrotic lung disease beyond idiopathic pulmonary fibrosis. Eur Respir Rev 2019; 28: 190022.

8 Cottin V. Treatment of progressive fibrosing interstitial lung diseases: a milestone in the management of interstitial lung diseases. Eur Respir Rev 2019; 28: 190109.

9 Caminati A, Lonati C, Cassandro R, et al. Comorbidities in idiopathic pulmonary fibrosis: an underestimated issue. Eur Respir Rev 2019; 28: 190044.

10 Weatherald J, Montani D, Jevnikar M, et al. Screening for pulmonary arterial hypertension in systemic sclerosis. Eur Respir Rev 2019; 28: 190023.

11 Elia D, Caminati A, Zompatori M, et al. Pulmonary hypertension and chronic lung disease: where are we headed? Eur Respir Rev 2019; 28: 190065.

12 Cadranel J, Canellas A, Matton L, et al. Pulmonary complications of immune checkpoint inhibitors in patients with nonsmall cell lung cancer. Eur Respir Rev 2019; 28: 190058. 